University of Nebraska - Lincoln

DigitalCommons@University of Nebraska - Lincoln

$2-1-2002$

\title{
High-energy ion generation in interaction of short laser pulse with high-density plasma
}

\author{
Y. Sentoku \\ Osaka University, 2-6 Yamada-oka, Suita, Osaka 565-0871, Japan
}

V. Y. Bychenkov

P.N. Lebedev Physics Institute, Russian Academy of Science, Moscow 117 924, Russia

K. Flippo

University of Michigan, Ann Arbor, MI

Anatoly Maksimchuk

University of Michigan, tolya@umich.edu

K. Mima

Institute for Laser Engineering, Osaka University, 2-6 Yamada-oka, Suita, Osaka 565-0871, Japan

See next page for additional authors

Follow this and additional works at: https://digitalcommons.unl.edu/physicsumstadter

Part of the Physics Commons

Sentoku, Y.; Bychenkov, V. Y.; Flippo, K.; Maksimchuk, Anatoly; Mima, K.; Mourou, G.; Sheng, Z. M.; and Umstadter, Donald, "High-energy ion generation in interaction of short laser pulse with high-density plasma" (2002). Donald Umstadter Publications. 76.

https://digitalcommons.unl.edu/physicsumstadter/76

This Article is brought to you for free and open access by the Research Papers in Physics and Astronomy at DigitalCommons@University of Nebraska - Lincoln. It has been accepted for inclusion in Donald Umstadter Publications by an authorized administrator of DigitalCommons@University of Nebraska - Lincoln. 


\section{Authors}

Y. Sentoku, V. Y. Bychenkov, K. Flippo, Anatoly Maksimchuk, K. Mima, G. Mourou, Z. M. Sheng, and Donald Umstadter 


\title{
High-energy ion generation in interaction of short laser pulse with high-density plasma
}

\author{
Y. Sentoku $^{1, *}$, V. Y. Bychenkov ${ }^{2}$, K. Flippo ${ }^{3}$, A. Maksimchuk ${ }^{3}$, K. Mima ${ }^{1}$, G. Mourou ${ }^{3}$, Z.M. Sheng ${ }^{1}$, \\ and D. Umstadter \\ ${ }^{1}$ Institute for Laser Engineering, Osaka University, 2-6 Yamada-oka, Suita, Osaka 565-0871, Japan \\ ${ }^{2}$ P.N. Lebedev Physics Institute, Russian Academy of Science, Moscow 117 924, Russia \\ ${ }^{3}$ Center for Ultrafast Optical Science, University of Michigan, Ann Arbor, MI 48109-2099, USA \\ *Corresponding author. Fax: 81-6/6877-4799; Email: sentoku@ile.osaka-u.ac.jp
}

\begin{abstract}
Multi-MeV ion production from the interaction of a short laser pulse with a high-density plasma, accompanied by an underdense preplasma, has been studied with a particle-in-cell simulation and good agreement is found with experiment. The mechanism primarily responsible for the acceleration of ions is identified. Comparison with experiments sheds light on the ion-energy dependence on laser intensity, preplasma scale length, and relative ion energies for a multi-species plasma. Two regimes of maximum ion-energy dependence on laser intensity, $I$, have been identified: subrelativistic, $\propto I$; and relativistic, $\propto \sqrt{I}$. Simulations show that the energy of the accelerated ions versus the preplasma scale length increases linearly and then saturates. In contrast, the ion energy decreases with the thickness of the solid-density plasma.
\end{abstract}

PACS 52.40.Nk; 52.35.Mw; 52.35.Nx; 52.25.Gj; 52.75.Di

\section{1}

\section{Introduction}

At focal intensities of $I>10^{18} \mathrm{~W} / \mathrm{cm}^{2}$, high-intensity laser-triggered ion acceleration results in the formation of a multi-MeV beam propagating in the forward direction [13]. The mechanisms and characteristics of ions triggered by the interaction of a short laser pulse with a plasma are of current interest because of their importance to novel-neutronsource development [4], isotope production [5, 6], fundamental nuclear physics at extremely short time scales [6], hadron therapy [7], relativistic ion-beam production [8], modeling of astrophysical phenomena in the laboratory [9], and the fast ignitor scenario with light ions [10].

It has long been understood [11] that ion generation in a laser-produced plasma relates to hot electrons. The commonly recognized effect responsible for ion acceleration is charge separation in the plasma due to high-energy electrons, driven by the laser inside the target $[1,3,12,13]$ and/or an inductive electric field resulting in the self-generated magnetic field [14], although a direct laser-ion interaction has been discussed for extremely high laser intensities $\sim 10^{22} \mathrm{~W} / \mathrm{cm}^{2}$ [8]. These electrons can be accelerated up to multi-MeV energies due to several processes, such as stimulated forward Raman scattering [15], resonant absorption [16], laser wake fields [17], ponderomotive acceleration by standing [18] and propagating [12] laser pulses, "vacuum heating" due to the $\boldsymbol{v} \times \boldsymbol{B}$ component of the Lorentz force [19] or the Brunel effect [20], wave-breaking of self-modulated laser wake fields or injection via wavebreaking of Raman-backscattered plasma waves [21], and betatron resonance provided by laser-pulse channeling [22]. Because of the plethora of mechanisms for electron acceleration and the corresponding electric-field generation, different regimes of ion acceleration are possible. Clearly, a better understanding of the mechanisms of forward ion acceleration in the interaction of a laser pulse with a solid target and quantification of the dependences of ion yield in terms of the laser pulse and the plasma parameters is essential for the success in new applications of laser-triggered ion beams.

Recent publications show that there is some controversy about the origin of the high-energy ions. The results obtained from some experiments $[5,13]$ provide evidence that the observed $\mathrm{MeV}$-ions were generated and accelerated in the plasma at the front of the target, conflicting with experiments $[3,23]$ that indicated that proton acceleration took place at the back of the target. The electrostatic model of ion acceleration suggests that the origin of the ions is the same for both of these experiments and that the only difference is in the plasma thickness: whether or not the plasma extends to the rear surface. We believe both situations are possible, depending on laser-pulse characteristics and foil-material conductivity. There is not only a controversy about the origin of the high-energy ions but also a dramatic disagreement on the hot-electron penetration in solid targets $[24,25]$. The latter experiments have been performed using green light of approximately the same pulse duration with laser intensities up to $4 \times 10^{18} \mathrm{~W} / \mathrm{cm}^{2}$ and $10^{19} \mathrm{~W} / \mathrm{cm}^{2}$, correspondingly. However, the penetration depth of hot electrons in [24] was more than two orders of magnitude longer than in [25]. Two possible disparate scenarios may explain these controversies in high-energy electron and ion generation.

Scenario 1 . When a solid target is highly resistive the return current within it is strongly suppressed. In this case hot electrons generated in the laser-plasma interaction in front of the foil penetrate only to a distance of their Debye length, $\lambda$ Dh These electrons are pulled back within the target (if the foil thickness exceeds the Debye length of hot electrons) by the electrostatic fields they set up and return in a fountain shape. 
The Debye sheath accelerates ions that appear to originate from the front of the target.

Scenario 2. Hot electrons penetrate deep inside the foil and reach the rear of the target. Such electrons can only propagate due to the return current [26, 27], which requires high conductivity within the target. Such a scenario corresponds to ion acceleration at the back of the target. Clearly, between these two disparate scenarios there might be an intermediate situation where the plasma comes into being in some other part of the target's thickness. Though, due to the laser prepulse, ionization by fast electrons, or electrical breakdown, the cold insulator might become a plasma conductor. The physical picture of this transformation arising in the bulk of the target is still incomplete. Note that [28] sheds some light on the latter issue, demonstrating a four times difference in the penetration depth of fast electrons in metals and insulators due to the effect of a self-consistent electric field, which pulls electrons back.

The crucial issue in the interaction of laser pulses with foil targets is preplasma formation before the peak of the pulse reaches the target. One may attribute such a preplasma as a reason for enhanced electron generation and, hence, enhanced electric-field generation which effectively accelerates the ions. For a low laser energy contrast ratio one may also expect an extension of the plasma to the rear surface of the foil target even before the main pulse's arrival. For instance, in [27], which models the transport of fast electrons generated by a 20-J laser, it was shown that only $0.6 \mathbf{J}$ are required to ionize a target of 250- $\mu \mathrm{m}$ thickness. Note that the experiment in [24] was performed with an order of magnitude higher laser energy (and so larger prepulse energy) than the experiment in [25], which displayed a dramatic difference in the hot-electron penetration depths. The aim here is to see how both underdense preplasma scale length and solid dense plasma thickness determine the efficiency of ion generation.

In this paper we report on the results of a fully relativistic two-dimensional (2D) particle-in-cell (PIC) simulation of multi$\mathrm{MeV}$ ion generation in the interaction of a short laser pulse with a dense plasma having a preplasma in front of it. We found that the theoretical dependences of the ion-generation efficiency versus laser intensity and plasma profiles agree well with recent experiments at the Center of Ultrafast Optical Science at laser intensities of $I \leq 6 \times 10^{18} \mathrm{~W} / \mathrm{cm}^{2}$ [5] and the data on maximum ion energies from the experiments performed at the Rutherford Appleton Laboratory on the Vulcan laser [13].

Because of the complexity of the physics involved, our use of PIC simulations is not intended to fully model all experiments but is used as a benchmark for interpreting the data most relevant to scenario 1 . In contrast to [26, 27], we do not model the physics inside the initially cold target; instead we assume a given solid density plasma slab whose back side is representative of the hot plasma-cold solid interface expected in a real target with low conductivity. Our simulations which self-consistently describe the laser-plasma interaction are subject to the further complication attendant with modeling electron-medium interaction within a cold target.

In a typical experiment on ion acceleration, a beam of protons rather than other ion species is observed in the forward direction, due to $\mathrm{H}_{2} \mathrm{O}$ contamination of the foil surface. Therefore, there is much interest in the simulation of ion ac- celeration from a multi-species plasma. In this context, with a PIC code, we examined in detail the high-energy ion characteristics from a two-component (hydrogen and deuterium) ion plasma.

In the present paper we describe the mechanism of ion acceleration through electron stochastic heating. We consider the transfer of laser energy to the electrons by the interaction of the incident and reflected electromagnetic waves in a preplasma with a strongly modulated density. We find that the hot-electron temperature, and so the fast ion energy, scales linearly with intensity at subrelativistic laser fields and as the square root of intensity at the relativistic limit.

The paper is organized as follows. Section 2 presents the results of the PIC simulation and a description of the electron heating. Such global ion characteristics as the energy cutoff and the emittance are discussed in Section 3. Section 4 reports on the comparison between experimental measurements and PIC simulations, and we conclude with a discussion and summary in Section 5.

\section{Particle acceleration from PIC simulations}

The 2D PIC simulations were performed for a 140fs linearly polarized laser pulse with a wavelength $\lambda=1 \mu \mathrm{m}$, normally incident onto an underdense plasma slab, having an exponential density profile with a spatial scale length $L=5$ $\mu \mathrm{m}$. There was a thin dense plasma slab behind it with a density 40 times higher than the critical density and a thickness $d=0.25 \mu \mathrm{m}$. This thickness is ten times larger than the skin depth, so the plasma slab is not transparent to the laser pulse. We use such a preplasma to model the blow-off plasma created by the laser prepulse which interacts with the foil before the main pulse reaches the target. The laser pulse with vacuum electric field amplitude $E_{y 0}$ propagates in the $X$ direction of an $X-Y$ simulation plane and has a 3- $\mu \mathrm{m}$ focal size. The normalized amplitude of the laser vector potential, $a=e E_{y 0} d$ $m c \omega_{0}$, where $m$ is the electron rest mass, $c$ is the light velocity, and $\omega_{0}$ is the laser frequency, was in the range $0.5 \leq a \leq$ 13.8, which corresponds to laser intensities from $3.3 \times 10^{17}$ to $2.5 \times 10^{20} \mathrm{~W} / \mathrm{cm}^{2} \mu \mathrm{m}^{2}$. The total simulation box is $50 \mu \mathrm{m}$ $\times 10 \mu \mathrm{m}$ and the dense plasma target is at $X=10 \mu \mathrm{m}$. A long vacuum region behind the target was placed to reduce boundary effects. For a laser intensity $a=2$, we performed simulations for different preplasma scale lengths, $0<L / \lambda<10$, and plasma target thicknesses, $0.1<d / \lambda<8$. A hydrogen-deuterium plasma was chosen with $20 \% \mathrm{H}$ and $80 \% \mathrm{D}$.

Although for the experiments performed on the pw-laser an amplified spontaneous emission (characterized by intensity contrast ratio $\sim 10^{-4}$ and occurring in the $n s$-period before the main pulse) is able to produce a preplasma with a scale length up to $40 \mu \mathrm{m}$ [3], our choice of a relatively thin plasma slab is motivated mainly by the TW-scale experiments with a higher contrast ratio and a shorter prepulse [5]. According to [25], for such experiments the temperature of bulk electrons in the solid plasma is around $1 \mathrm{keV}$. Initially only a thin skin layer, less than $0.1 \mu \mathrm{m}$, is heated to this temperature. During the heating, the size of the hot dense plasma increases due to the energy transport of the bulk electrons inside the target. However, the 
heat-transport velocity, $\sim f v_{\mathrm{Te}}$, where $v_{\mathrm{Te}}$ is the electron thermal velocity and $f$ is the heat flux inhibition factor, is rather low, and, for a subpicosecond laser pulse, a hot dense plasma cannot expand to greater than a micron size. For example, at a pulse duration of $400 \mathrm{fs}$ [5] and a commonly used value of $f \sim$ 0.1 , one may estimate $d \sim 1 \mu \mathrm{m}$. This is the motivation for the choice of a relatively thin dense plasma slab. For the laser intensities considered here, the main laser energy is converted to hot electrons, which may penetrate at least to their Debye length. For instance, for a hot-electron density of about $n_{\mathrm{c}}$ and a temperature $\sim 1 \mathrm{MeV}$, it is $\sim 0.3 \lambda$. As was explained in Section 1, we follow scenario 1 for hot-electron penetration considering the high-resistivity material, where the right plasma boundary is representative of the hot plasma-cold solid interface. This is likely to be relevant to experiments [5] with insulators. The measurements of the hot-electron penetration depth [25] for the same typical parameters as in the experiments [5] have shown a rather short penetration depth $<3 \mu \mathrm{m}$. In order to model deeper energy deposition, for example due to a prepulse, we changed $d$ in our simulations by up to $8 \lambda$.

A brief picture of ion acceleration has been proposed [3, 5, $10,29]$. The electrons, accelerated in the under-dense plasma (with density, $n_{\mathrm{e}}$, less than critical, $n_{\mathrm{c}}$ ), penetrate deep inside the target, or even through it, and set up a strong electrostatic field (Debye sheath) which accelerates ions forward and decelerates electrons. Thus, the kinetic energy of the fast electrons transforms into the electrostatic field energy and the electric potential, which accelerates ions and is expected to be at the level of the hot-electron energy. Correspondingly, the bulk ion energy scales as the hot-electron energy, although the maximum ion energy might be significantly higher.

The above-mentioned model suggests that the generation of high-energy electrons is crucial to ion acceleration. At the same time, electron motion is very complicated because of the combined effect of the pump, self-generated fields, and plasma inhomogeneity. In Figure 1 we show a 2D distribution of the electromagnetic energy, the magnetic field $\left(B_{z}\right)$, and the longitudinal electrostatic field $\left(E_{x}\right)$ for a subrelativistic laser intensity, $a=1$. Reflection of laser light produces a standing wave, which is seen in Figure 1a to be a modulation of the laser intensity with the scale $\lambda / 2$. Laser-beam focusing is insignificant in the underdense plasma. Surface currents and the backward-expelled electrons cause the so-called "fountain effect" - generated magnetic field with a maximum up to $40 \%$ of the laser field (Figure 1b).

Good correlation with the laser-intensity modulations of the longitudinal electric field in the preplasma can be seen clearly in Figure 1c. We have concluded that the latter is due to the ponderomotive effect at each maximum of the laser intensity. However, in an arbitrary $Y$-slice, the electrostatic field demonstrates chaotic behavior (Figure 1d) that cannot be seen in Figure 1c because of the rather small scale of the electrostatic fluctuations across the laser beam. On the other hand, averaged over the laser focal size, this electrostatic field shows a well-defined periodic structure in the $X$-direction with a wavelength of $\lambda / 2$. The electron-plasma density has similarly strong modulations. Hence, the acceleration of the electrons occurs in a preplasma with a very complicated electromagnetic-field structure and density. Propagating through the target, these electrons produce a strong charge-separation field which reaches $40 \%$ of the laser field behind the target and smoothly decreases with distance as shown in Figure 1d.

The PIC model gives details of the electron-energy distribution as shown in Figure 2c for $a=1$. The phase-space plots (Figure 2a, b) show space modulations with scale $\lambda / 2$ due to the formation of a standing electromagnetic wave, which plays an important role in electron heating. For this run, $60 \%$ of the laser energy is reflected, namely, the reflectivity coefficient related to the field amplitude is $r \sim 0.8$. The amplitude of the longitudinal momentum oscillations due to the formation of a standing electromagnetic wave can be estimated as $p_{\chi} / m c$ $\sim(1+r)^{2} a^{2} \sim 3$ (cf. Figure $2 b$ ). During several laser cycles, the electron motions become stochastic. They penetrate deep inside the target, mainly due to the $\boldsymbol{v} \times \boldsymbol{B}$ accelerating force, acquiring energy from the electromagnetic field.

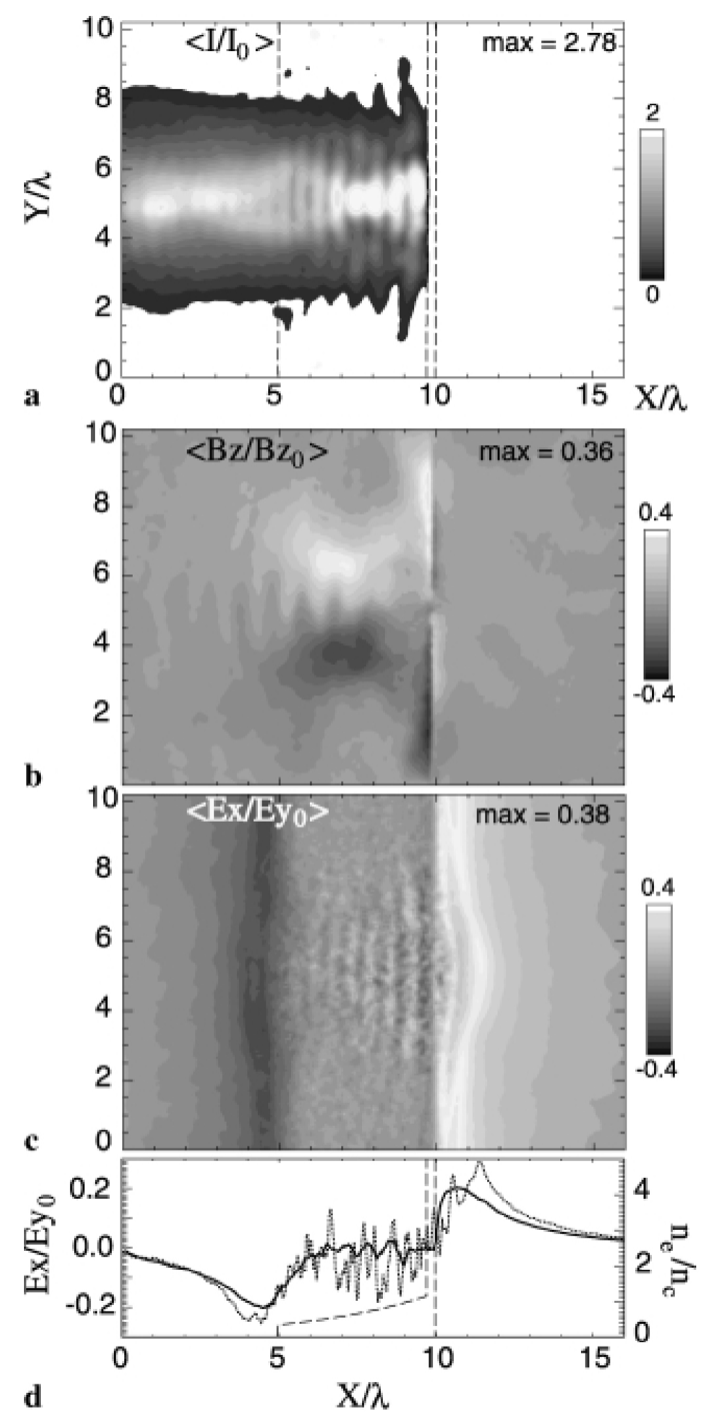

Figure 1 The electromagnetic energy at $106 \mathrm{fs}$ (a), the quasistatic magnetic field at $185 \mathrm{fs}(\mathbf{b})$, the longitudinal electrostatic fields at $185 \mathrm{fs}$ (c), and corresponding slice plot at $Y=5 \mu \mathrm{m}(\mathbf{d})$ for dimensionless laser field $a=1$. The dashed lines indicate the initial plasma boundaries. All plots are time-averaged over the laser period. 

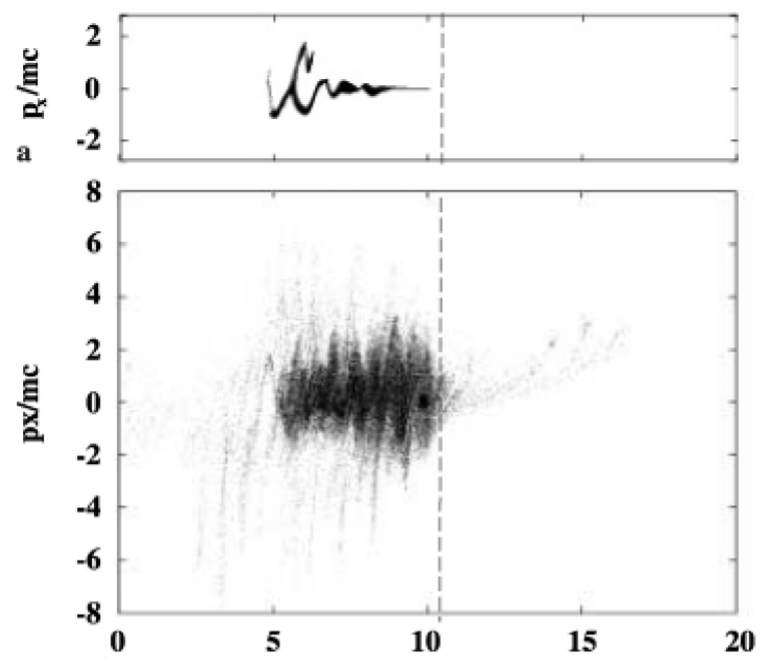

b

$\mathbf{X} / \lambda$

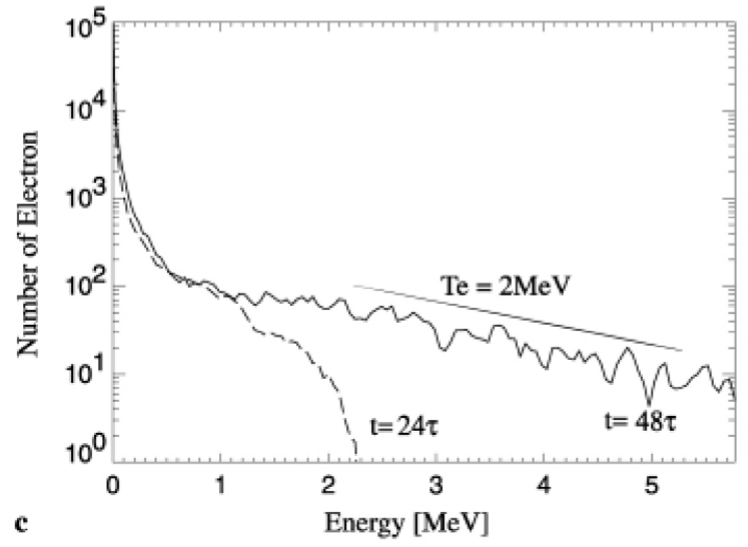

Figure 2 The electron phase-space plots $X-p_{x}$ during the laser pulse, at $t=$ $40 \mathrm{fs}$ (a)and $t=80 \mathrm{fs}(\mathbf{b})$. The energy distribution of forward-accelerated electrons (c) during the laser pulse, at $t=80 \mathrm{fs}$, and after it, at $t=160 \mathrm{fs}$. Laser intensity corresponds to $a=1$.

We considered a model problem of electron motion in a homogeneous underdense plasma with an electromagnetic field approximated by a uniform incident and counter-propagating (reflected) plane waves

$E_{y 0}=E_{0}(t) \cos (\omega t-k x)+r E_{0}(t) \cos (\omega t+k x)$,

$B_{z 0}=k E_{0}(t) \cos (\omega t-k x)-k r E_{0}(t) \cos (\omega t+k x)$,

with the amplitude corresponding to $a=0.5,1$, and 2 and $r=$ 0.75. In the above PIC simulation, the electrostatic field was chosen as

$E_{x}=\alpha E_{0}(t) \sin (2 k x)$,

with $\alpha=0.2$. The wave amplitude, $E_{0}(t)$, increases exponentially as a function of time, $E_{0}(t)=\min \{1, \exp [(t-T) / T]\}$, until saturation at $t=T=20 \tau(\tau=2 \pi / \omega)$.

Calculations are done for 2000 electrons in a plasma with $n / n_{\mathrm{c}}=0.3$ during $200 \tau$. Figure 3 shows the electron kinetic energy, $\gamma-1$, versus the phase, $\varphi$, for different laser intensities, where $\varphi=\omega t-k x$. The maximum electron energy increases as $a^{2}$ at $a \lesssim 1$ and has a somewhat weaker dependence at $a>$ 1 . The energy dependence on laser intensity from this model

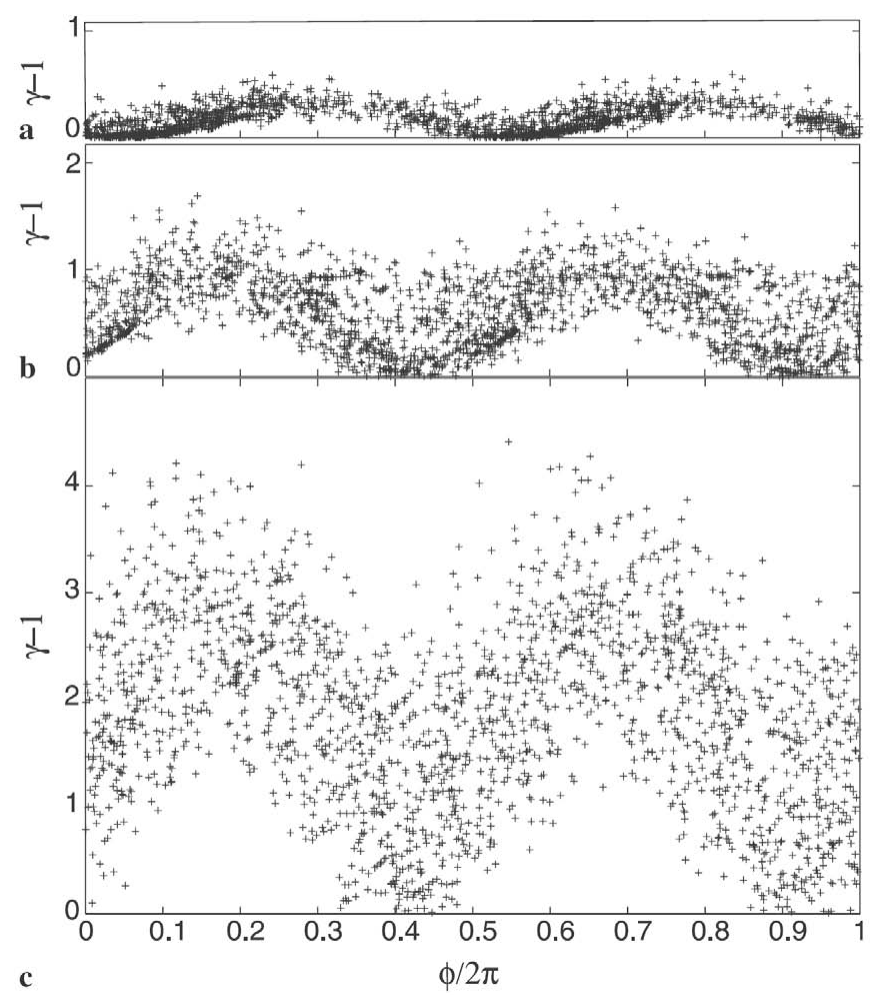

Figure 3 The electron-energy plots in a system of two counter-propagating waves at $t=50 \tau$ for $a=0.5(\mathbf{a}), 1(\mathbf{b})$, and 2(c).

problem qualitatively agrees with the result of the PIC simulation. In the course of time the electron motion takes on a chaotic character. To study stability of the system we switch on small electron-momentum perturbations, $\delta \boldsymbol{P}$, at some time $t_{0}$. The difference of single-particle orbits with and without perturbations demonstrates the stochastic behavior of the system. Namely, $\delta P_{x}=0.0001 \mathrm{mc}$ was added at $t=t_{0}=50 \tau$. Figure 4a and $\mathrm{b}$ show the orbits of an electron in $p_{x}-p_{y}$ space in the case $a=1$ and $r=0.1$ without and with the perturbation, respectively. There is no significant difference in either plot.

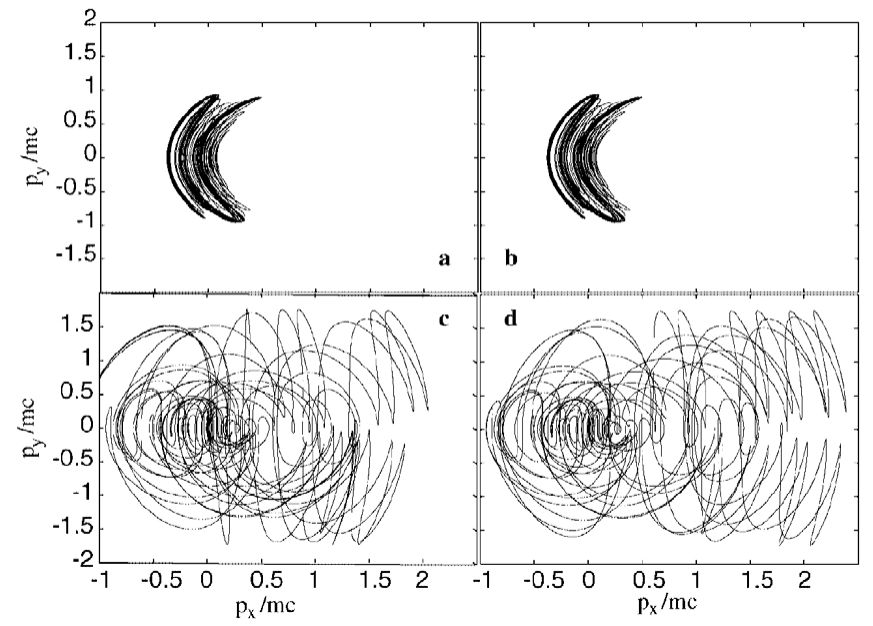

Figure 4 The electron orbit in phase space $p_{x}-p_{y}$ for $r=0.1$ (a), and $r$ $=0.75$ (c), in a two counter-propagating-wave system. The perturbation was switched on at $t=50 \tau$, which results in transformation of the orbits $\mathbf{a}$ and $\mathbf{c}$ to $\mathbf{b}$ and $\mathbf{d}$, correspondingly. Computations performed for $a=1$. 
When the reflection coefficient is increased to $r=0.75$, this is no longer the case, as shown in Figure $4 \mathrm{c}$ and d, with and without perturbation, correspondingly. To clarify this the following Lyapunov exponents have been calculated [30]:

$$
\lambda=\lim _{\Delta t \rightarrow \infty} \frac{1}{\Delta t} \sum \log \frac{\left|\boldsymbol{P}\left(\boldsymbol{P}_{\mathbf{0}}+\delta \boldsymbol{P}\right)-\boldsymbol{P}\left(\boldsymbol{P}_{\mathbf{0}}\right)\right|}{|\delta \boldsymbol{P}|},
$$

where $\boldsymbol{P}_{0}$ is the momentum just before the perturbation was added (at $50 \tau$ ), $\Delta t$ is the time passed after that, and the summations are over the time steps. For negative Lyapunov exponents the system is stable with respect to small perturbations. This means that two particles with and without perturbation stay very close in phase space. However, if the Lyapunov exponent is positive their orbits become dramatically different, proving that the system is chaotic. The calculated Lyapunov exponents are shown in Figure 5. From this figure one may conclude that the $r=0.1$ case is stable with respect to momentum perturbation, but the $r=0.75$ case is unstable, i.e. the system is chaotic and electrons experience stochastic heating. The bifurcation occurs somewhere around $r=0.2$.

In Figure 6, the hot-electron temperature extracted from the PIC simulations, $T_{\mathrm{h}}$, at the solid plasma surface is plotted

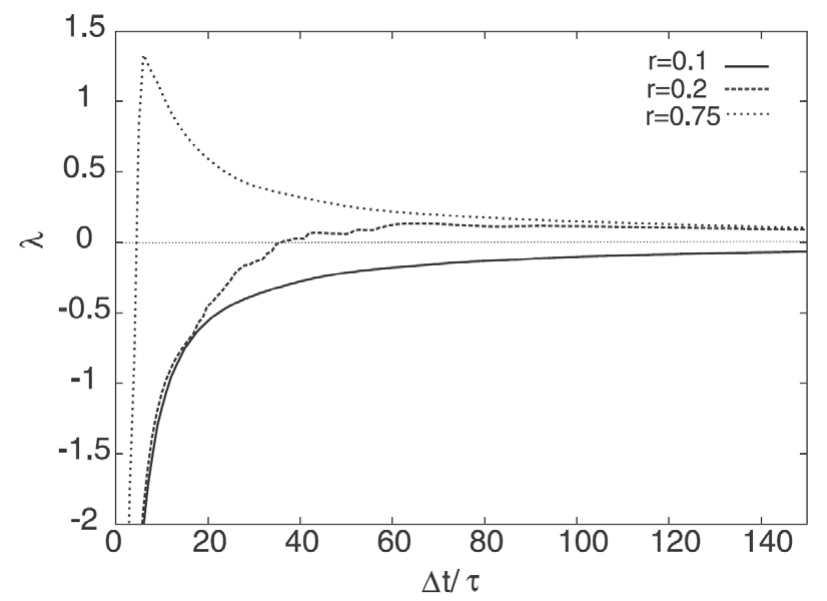

Figure 5 The Lyapunov exponents of the electron motion in a two counterpropagating-wave system versus the time elapsed after the perturbation was switched on.

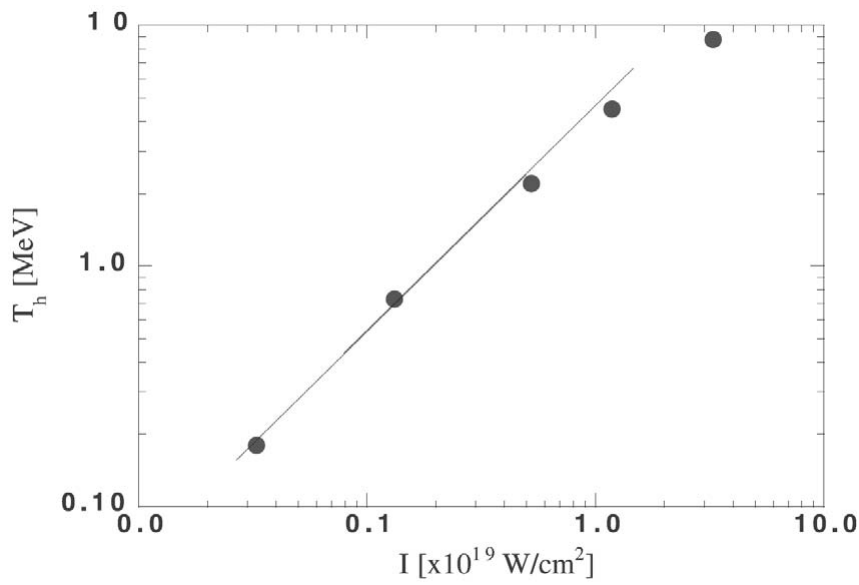

Figure 6 Hot-electron temperature at the solid plasma surface versus the laser intensity at $80 \mathrm{fs}$. versus the laser intensity. It is proportional to the laser intensity in the subrelativistic case and has a significantly weaker dependence, close to the square root, at $a \gg 1$.

The hot electron temperature dependences of $\propto I$ and $\propto \sqrt{I}$ qualitatively correspond to electron-energy gain in the electromagnetic field propagating in the plasma with $c k<\omega$. The dependence of this gain $\gamma-1$ on laser intensity can be estimated from the known integrals [31] of electron motion in an electromagnetic field:

$p_{x} / m c=(c k / \omega)(\gamma-1)$,

$p_{y} / m c=a$,

$\gamma=\left(1+p_{x}^{2} / m^{2} c^{2}+p_{y}^{2} / m^{2} c^{2}\right)^{1 / 2}$,
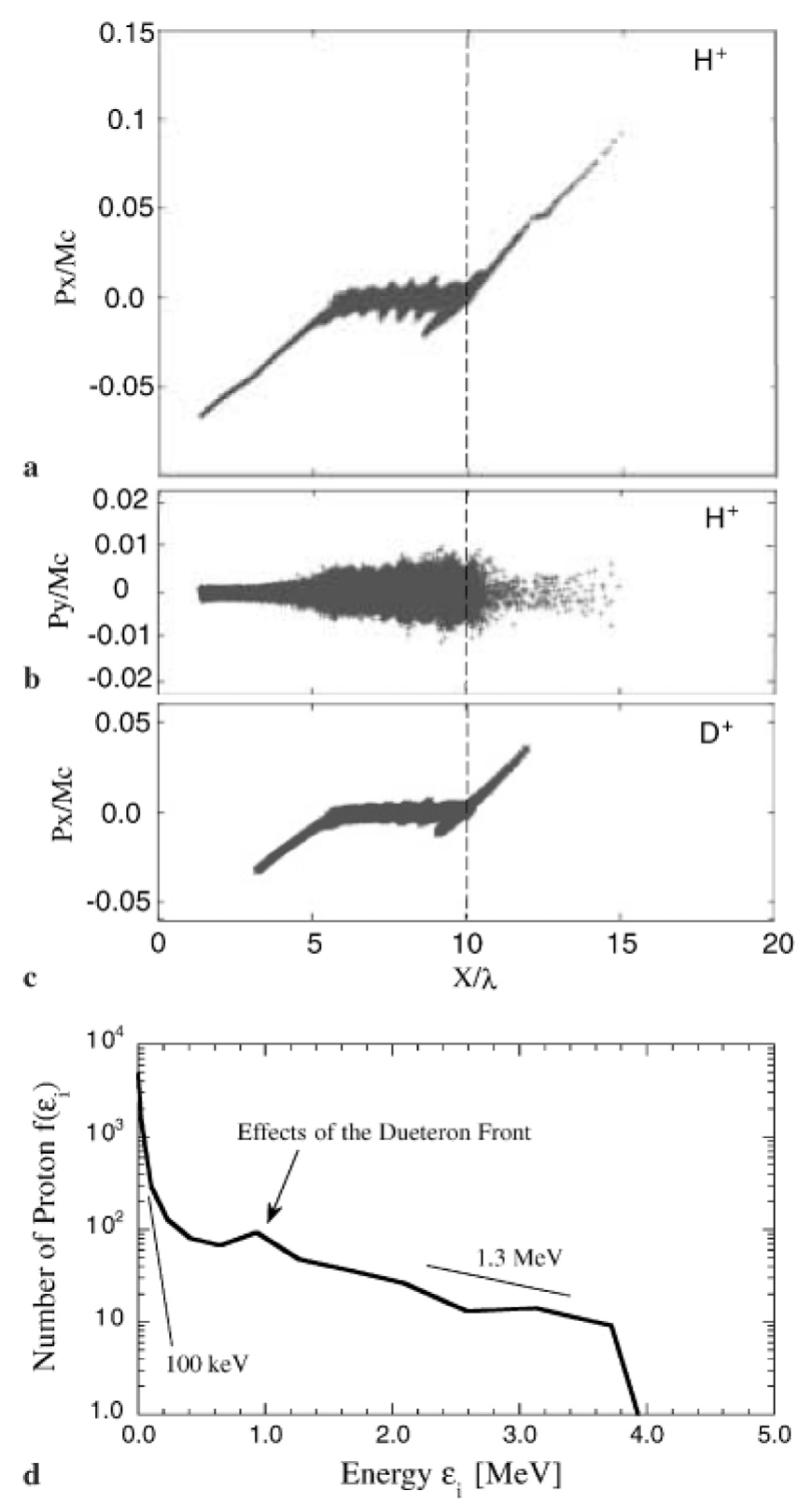

Figure 7 The simulation results for $a=1$ : the ion phase-space plots $X-p_{x}$ (a) and (c) for $\mathrm{H}^{+}$and $\mathrm{D}^{+}$ions, correspondingly, and $X-p_{y}$ (b) for $\mathrm{H}^{+}$ions at 330 fs. The energy distribution of forward-accelerated protons is shown in (d) at $t$ $=330 \mathrm{fs}$. Dashed vertical line shows the position of a thin overdense plasma slab, and $M$ is the ion mass, for protons and deuterons, respectively. 
which are written for an electron initially at rest and correspond to the Landau solution in a vacuum [32] at $c k=\omega$. According to (4), $\gamma-1=a^{2} / 2 \propto I$ at $a^{2} \ll \omega^{2} /\left(\omega^{2}-c^{2} k^{2}\right)$ and $\gamma-1=a \omega /\left(\omega^{2}-c^{2} k^{2}\right)^{1 / 2} \propto I^{1 / 2}$ at $a^{2} \gg \omega^{2} /\left(\omega^{2}-c^{2} k^{2}\right)$. Such a scaling is similar to that obtained from the ponderomotive potential [18] $T_{\mathrm{h}}=m c^{2}\left(\left(1+\mathrm{a}^{2}\right)^{1 / 2}-1\right)$. However, in contrast to the scaling of [18], it includes the important contribution of the longitudinal electron momentum, which cannot be considered negligible compared to the electron transverse momentum. Note that an electron interacting with a relativistically strong electromagnetic field in a plasma acquires an energy $m c^{2} a$, but not $\sim m c^{2} a^{2}$, which follows from the vacuum solution [32]. Clearly, the value of the wave number is somewhat indefinite in a inhomogeneous preformed plasma strongly coupled with the laser field, $0<k<\omega / c$. Nevertheless, the estimation of electron temperature, $T_{\mathrm{h}}=\gamma-1$, using $1-c k / \omega \sim$ 1 agrees with the order of magnitude of our PIC simulations and gives the characteristic value of the charge-separation potential, $e \varphi \sim T_{\mathrm{h}}$, which accelerates the ions.

The PIC simulations suggest that the preplasma, which can be generated due to the finite intensity contrast ratio of the laser pulse, makes the laser capable of producing ions in the $\mathrm{MeV}$ range at $I>5 \times 10^{17} \mathrm{~W} / \mathrm{cm}^{2}$. In Figure 7 (a,b) we show longitudinal and transverse proton momenta versus distance $X$. Comparison between panels a and $\mathrm{b}$ demonstrates that the high-energy protons are expelled as two beams in forward and backward directions, and the energy of forward-accelerated ions is higher. The energy of deuterons is $4-5$ times lower than protons (Figure 7c). Deuterons are two times heavier than protons and have less mobility. Following behind the protons, they experience a significantly lower accelerating electric field and, therefore, gain less energy.

Figure 7d shows the ion-energy spectrum for $a=1$. It demonstrates the low-energy (100-200 keV) ion bulk, and the hot-ion tail (1-1.5 MeV) which smoothly transforms to a flat distribution with a sharp energy cutoff $(4 \mathrm{MeV})$. Such a flat distribution with energy cutoff is typical for an electrostatic mechanism of ion acceleration [1-3, 13, 33, 34]. It corresponds to the maximum electric potential produced by the hot electrons. One more piece of evidence for the electrostatic nature of ion acceleration is provided by the peak in the proton distribution at energy of $\sim 1 \mathrm{MeV}$, which corresponds to the front of the deuterons and defines in turn the self-consistent accelerating field.

\section{Global characteristics of accelerated ions}

The curve in Figure 8 shows the simulation results of the maximum ion energy versus laser intensity. This dependence is close to $\propto I$ for subrelativistic intensities. However, at relativistic laser intensities the maximum ion energy displays a weaker dependence on $I$, somewhat like $\propto \sqrt{I}$. Note that without a preplasma the maximum ion energy scales $\propto$ $a$ [1]. Comparison between Figure 8 and Figure 6 shows that the characteristic ion energy, $\varepsilon$, is proportional to the electron temperature

$\varepsilon \simeq \beta T_{\mathrm{h}}$,

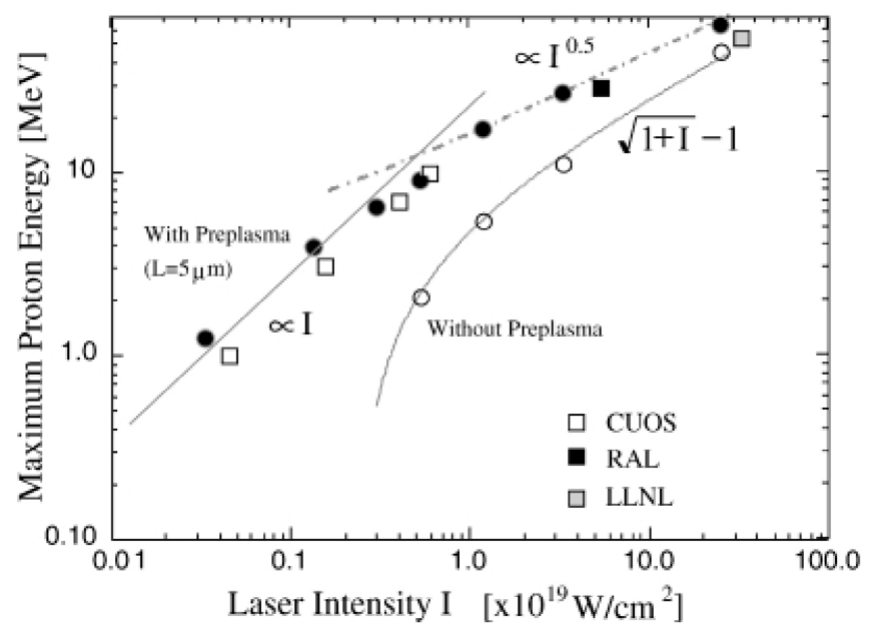

Figure 8 The intensity dependence of the maximum energy of protons (black dots) in comparison with the experimental data (squares): CUOS [5], RAL - [13], and LLNL - [3], and simulations without preplasma (open circles).

where for thin targets $\left(d \lesssim \lambda_{\mathrm{Dh}}\right) \beta \simeq 4$ is similar to the result of [11], where the bulk ion energy scaled as $4.5 T_{\mathrm{h}}$. Equation (5) reflects the commonly recognized fact that ion generation in a laser-produced plasma relates to the hot electrons. However, the dependence of ion energy on $a$ for subrelativistic intensities is different from the $\propto a^{8 / 9}$ fit proposed in [11] for the low-intensity domain of values of $a$ from $10^{-4}$ to 0.8 .

The PIC simulation strongly suggests that the preplasma significantly enhances the production of the high-energy ions as compared to the case of an overdense plasma with a sharp boundary, which is typical for a high contrast ratio. This enhancement is most pronounced at moderate laser intensities, $a$ $\sim 1$, and is significantly less for ultrarelativistic intensities. The ratio of maximum ion energy to that without a preplasma drops from 4.5 at $a=1$ to 1.4 at $a=14$. Therefore, at laser intensities of current interest, $I=10^{19}-10^{20} \mathrm{~W} / \mathrm{cm}^{2}$, there is direct evidence of a significant increase of ion energy due to preplasma.

From the study of ion trajectories, we conclude that the maximum energy gain is obtained when the protons are accelerated from the back side of the overdense plasma, in accordance with the spatial distribution of the electrostatic field (Figure 1d). Indeed, the electric field is maximum behind the dense plasma slab so that the rear-side protons respond first and the others that follow behind them are accelerated by a screened potential. However, this result needs to be reconciled with the contradictory experimental observations of ion acceleration from either the front $[1,13]$ or rear surfaces [3]. For the experiments with high intensity contrast ratio $[1,5]$, the rear surface of solid dense plasma is, in fact, the front surface of a foil because the thickness of the highly ionized region is negligible as compared to the foil thickness. This is likely not the case for the experiment in which a rear-surface origin was found [3], where a very intense prepulse preceded the main laser pulse.

We have investigated the ion-energy dependences on the preplasma scale length and the solid density plasma thickness. The corresponding figures, Figures 9 and 10, show these dependences for $a=2$. The maximum ion energy was found to increase with the preplasma scale length and then to saturate. 


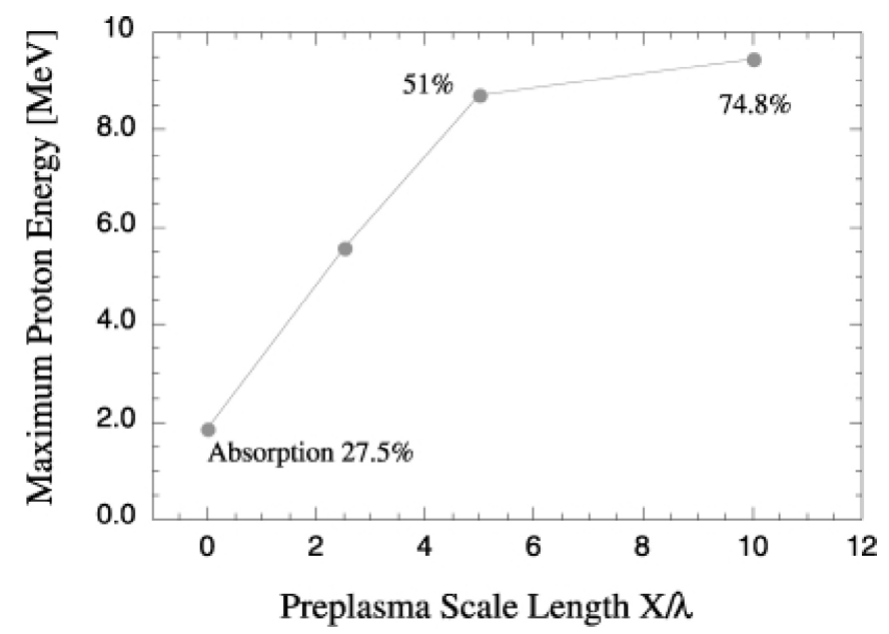

Figure 9 The maximum proton energy versus the preplasma scale length for $a=2$.

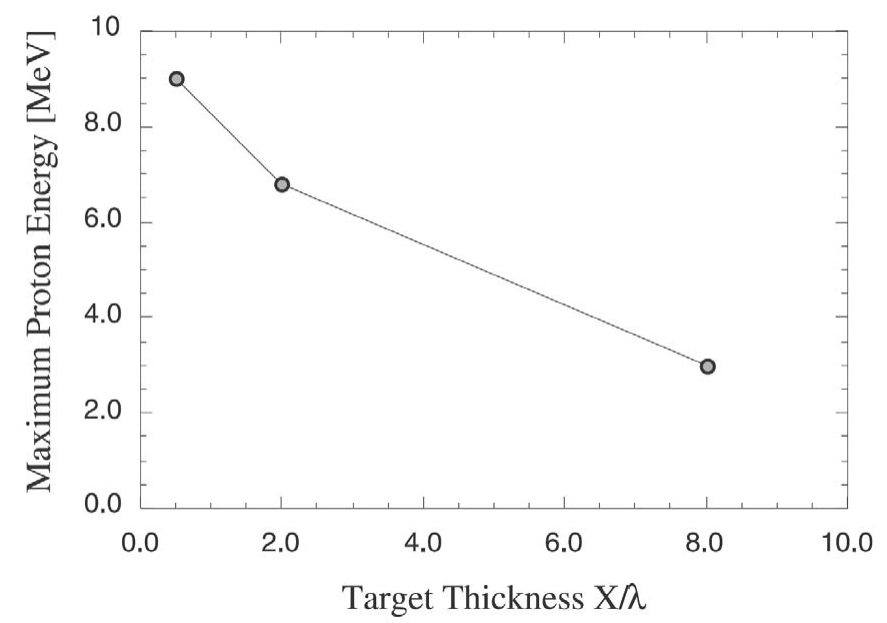

Figure 10 The maximum proton energy versus the overdense plasma thickness for $a=2$.

The absorption coefficient has a similar behavior. Hence, the ideal intensity contrast ratio is not optimal for efficient ion acceleration. Certainly, this "plateau" regime is restricted by some large enough scale length until all the laser energy dissipates and scatters in the preformed plasma.

In contrast, the maximum ion energy decreases as the target plasma thickness increases. We attribute this to a reduction in the averaged density, $n_{\mathrm{h}}$, of hot electrons, which accelerates ions. The electrostatic potential at the right boundary where the acceleration of the ions predominantly occurs scales as $n_{\mathrm{h}}$, namely e $\varphi \sim\left(n_{\mathrm{h}} / n_{\mathrm{c}}\right) T_{\mathrm{h}}$. If the number of accelerated electrons, $N_{\mathrm{h}}$, does not depend on the target thickness, the magnitude of the averaged hot-electron density decreases with the plasma thickness as $d^{-1}$. Actually, there were no significant differences of $N_{\mathrm{h}}$ and $T_{\mathrm{h}}$ with the different thick targets in the simulations. However, the dependence $n_{\mathrm{h}} \propto d^{-1}$ is not the case if the plasma thickness is small or large enough. Indeed, for $d$ $<\lambda_{\text {Dh }}$ the averaged density does not depend on $d$ because hot electrons circulate in the region of a typical size $\sim \lambda_{\text {Dh }}$, so that $n_{\mathrm{h}} \propto \lambda_{\mathrm{Dh}}^{-1}$. From this simple qualitative picture it follows that an increase in the overdense plasma size should result in an ion-energy drop when the plasma thickness reaches $d \approx \lambda_{\mathrm{Dh}}$ : $n_{\mathrm{h}} \approx N_{\mathrm{h}} / \lambda_{\text {Dh }} \approx$ const., if $d<\lambda_{\text {Dh }}$, and $n_{\mathrm{h}} \approx N_{\mathrm{h}} / d$, if $d>\lambda_{\mathrm{Dh}}$. Certainly this is valid if the hot electron energy losses are small in the dense plasma. For the plasma layer thickness used in our simulation these losses never exceeded 10\%-15\%. Otherwise, the scaling proposed may experience an additional smooth decline.

To further illustrate the ion-acceleration process we evaluated the emittance [35] of the proton beam at the back side of an overdense plasma slab. The emittance grows as the acceleration begins and then saturates. For moderate laser intensities, $a \sim 1$, we estimate the emittance as $\sim 2 \pi \mathrm{mm}$ mrad (5mm mrad for $a=1$ and $6 \mathrm{~mm} \operatorname{mrad}$ for $a=2$ ).

\section{$4 \quad$ Comparison with experiments}

Our simulations agree well with some features of the high-energy ion emission observed in the experiments performed with thin-film targets $[1,5]$. In [1], there was reported the existence of an optimum in laser prepulse intensity. This intensity was varied from $10^{-4}$ to 0.1 of the main pulse intensity of $10^{18} \mathrm{~W} / \mathrm{cm}^{2}$ in the experiments with a 400 -fs green-light pulse obtained from doubling the 1.053- $\mu \mathrm{m}$ light of a hybrid Ti: sapphire/Nd:phosphate chirped-pulse-amplified laser. The maximum proton energy shown by the experimental points in Figure 11 increases with the prepulse intensity for a contrast ratio $\lesssim 10^{-3}$ and then decreases. This is in qualitative agreement with the simulation results demonstrated in Figs. 9 and 10. It is likely that at rather low intensity $I \sim 10^{18} \mathrm{~W} / \mathrm{cm}^{2}$ the scale length of the preformed underdense plasma grows with the intensity of the prepulse without a significant increase in the volume of the overdense plasma if the prepulse intensity remains low enough. Accordingly, the maximum ion energy should increase and then saturate (Figure 9). However, at higher prepulse intensities, one might expect enhanced solid-density plasma production before the main laser pulse reaches the target. As a result, the efficiency of the ion acceleration decreases (Figure 10). The interplay between these two effects should result in a dependence similar to the experimental one shown in Figure 11 with the maximum at some moderate contrast ratio.

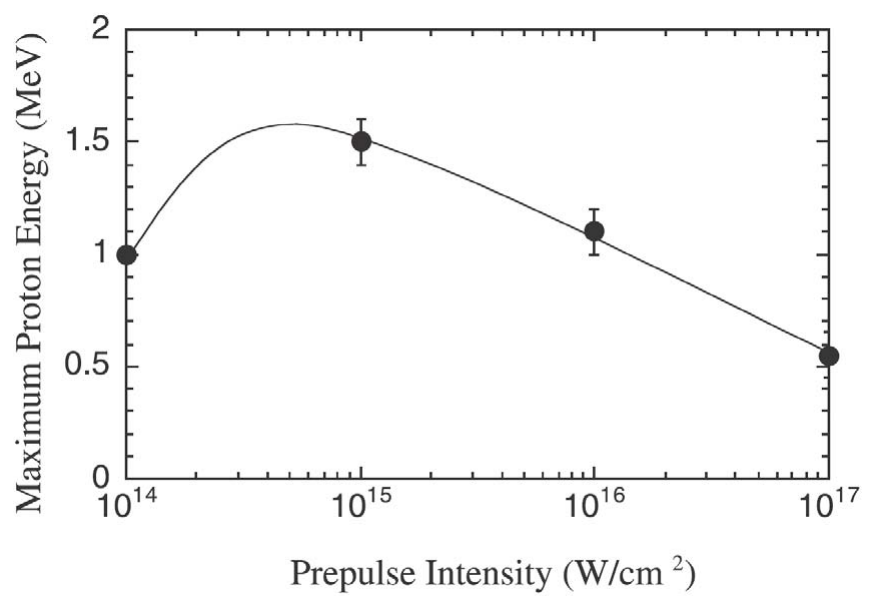

Figure 11 The measured maximum proton energy versus the intensity contrast ratio of an artificially produced prepulse with the time delay of $50 \mathrm{ps}$ in front of the main pulse. 
Our simulations quantify the inhibition of deuteron energy when they are accelerated along with protons. This effect was observed in an experiment [5] performed using a 4-J, 400-fs pulse of a Ti:sapphire/Nd:phosphate laser at the fundamental wavelength with an intensity contrast ratio of $5 \times 10^{5}$ : 1 . The experimental data on the activation of a boron sample with deuterons at $I=6 \times 10^{18} \mathrm{~W} / \mathrm{cm}^{2}$ suggested that their energy was $\sim 2 \mathrm{MeV}$, while the proton energy was up to $10 \mathrm{MeV}$. This is close to the simulation result that gives a deuteron energy four times less than the proton energy.

The maximum proton energy at $I=0.5-6 \times 10^{18} \mathrm{~W} / \mathrm{cm}^{2}$ was approximately proportional to the laser intensity [5]. Corresponding experimental data are shown in Figure 8 (open squares). The simulation results elucidate a $\propto I$ ion-energy dependence on the laser intensity observed in the experiment. At higher intensities our simulations predict the dependence somewhat close to $\propto \sqrt{I}$ that was also inferred from other experimental measurements [2]. We have found that the proton energy measured at the Rutherford Appleton Laboratory on the Vulcan laser [13] corresponds well to this prediction (black squares in Figure 8). In Figure 8 we also marked the ion energy (gray squares) which corresponds to measurements performed in the Lawrence Livermore National Laboratory on a petawatt laser [3] at the intensity $3 \times 10^{20} \mathrm{~W} / \mathrm{cm}^{2}$. For such intensity our simulations predict a higher ion energy. We believe that this difference might be attributed to a significant power in the prepulse of the petawatt laser, which is able to ionize a large volume of the solid target and hence reduce the efficiency of the ion generation.

\section{$5 \quad$ Conclusion}

In this paper, we have performed PIC simulations of ion acceleration triggered by a high-intensity short laser pulse interacting with a preformed plasma in front of an overdense plasma slab and have shown that the presence of underdense plasmas is crucial to high-energy ion generation. The results give evidence that a preplasma of several wavelengths size leads to enhanced generation of hot electrons, which penetrate into the target and, hence, produce a high charge separation electric field, effectively accelerating the ions from the dense plasma. Such a preformed plasma likely appears in most experiments because of the non-ideal shape of the laser pulse.

From the simulations described here, we inferred a dependence of the maximum ion energy on laser intensity. These simulations explain the measured linear dependence $\varepsilon_{\max } \propto I$ [5] for moderate laser intensities and the $\sqrt{I}$ dependence for high intensities, suggested in [2]. This scaling predicts $1-\mathrm{GeV}$ protons at $I=5 \times 10^{22} \mathrm{~W} / \mathrm{cm}^{2}$. However, our PIC simulation for such ultrahigh intensities shows an even higher proton energy, which corresponds to a dependence somewhat between $\sqrt{I}$ and $I$ for $I>10^{22} \mathrm{~W} / \mathrm{cm}^{2}$. Actually, in such an ultrarelativistic regime $\left(I>10^{22} \mathrm{~W} / \mathrm{cm}^{2}\right)$ one may expect such a sharpening of energy scaling because of additional acceleration mechanisms due to the inductive electric fields [14].

The physical picture behind these $I$ and $\sqrt{I}$ dependences is quite simple and is based on hot-electron generation in preformed plasma. The electrons interacting with a fast electromagnetic wave, $\omega>c k$, acquire an energy $m c^{2}(\gamma-1)$, which scales as $m c^{2} a^{2}$ and $m c^{2} a$ for subrelativistic and ultrarelativistic laser intensities, respectively. In fact, electrons experience a rather complicated stochastic motion in an inhomogeneous underdense plasma with self-consistent quasistatic fields and a reflected electromagnetic wave. We conclude that the formation of a standing electromagnetic wave is a key process leading to stochastic electron heating. During such heating, a quasithermal electron energy distribution forms with an effective temperature $T_{\mathrm{h}}$, which scales as $m c^{2}(\gamma-1) \propto \min \left\{a^{2}, a\right\} m c^{2}$. Hence, both the electrostatic potential of the charge-separation field and the ion energy have the same dependences on laser intensity. A recent experiment performed with subrelativistic intensities bears out the linear dependence of the maximum ion energy on laser intensity [5], while another [2] bears out the predicted square-root dependence.

In this paper, we described the influence of both the preformed and the overdense plasma sizes on ion generation. The observed decrease of ion energy with the overdense plasma thickness is due to the lowering of the averaged density of hot electrons, which accelerates ions. This effect can be significant for laser pulses having a powerful prepulse (such as from the petawatt laser), which is able to heat a thick domain of the target before the main laser pulse reaches it. We predict the saturation of ion energy when the solid plasma thickness exceeds the laser-pulse duration if hot-electron losses in the target are small. The observed increase of the maximum ion energy with the preplasma thickness and its saturation demonstrate that an ideal laser pulse with a very high contrast ratio is also not optimal for effective high-energy ion generation. The latter appears to be a result of the suppression of hot-electron generation in a short-density-scale-length under-dense plasma.

\section{Acknowledgements}

The work was partly supported by the Russian Foundation for Basic Research (Grant No. 00-02-16063) and the U.S. National Science Foundation. Y. S. would like to thank the computational staffs of ILE, Osaka University for their support. All numerical simulations were performed on a Hitachi-SR8000 at ILE.

\section{References}

1 A. Maksimchuk, S. Gu, K. Flippo, D. Umstadter, V.Y. Bychenkov: Phys. Rev. Lett. 84, 4108 (2000)

2 E.L. Clark, K. Krushelnick, M. Zepf, F.N. Beg, M. Tatarakis, A. Machacek, M.I.K. Santala, I. Watts, P.A. Norreys, A.E. Dangor: Phys. Rev. Lett. 85, 1654 (2000)

3 S.P. Hatchett, C.G. Brown, T.E. Cowan, E.A. Henry, J.S. Johnson, M.H. Key, J.A. Koch, A.B. Langdon, B.F. Lasinski, R.W. Lee, A.J. MacKinnon, D.M. Pennington, M.D. Perry, T.W. Phillips, M. Roth, T.C. Sangster, M.S. Singh, R.A. Snavely, M.A. Stoyer, S.C. Wilks, K. Yasuike: Phys. Plasmas 7, 2076 (2000); R.A. Snavely, M.H. Key, S.P. Hatchett, T.E. Cowan, M. Roth, T.W. Phillips, M.A. Stoyer, E.A. Henry, T.C. Sangster, M.S. Singh, S.C. Wilks, A.J. MacKinnon, A. Offenberger, D.M. Pennington, K. Yasuike, A.B. Langdon, B.F. Lasinski, J. Johnson, M.D. Perry, E.M. Campbell: Phys. Rev. Lett. 85, 2945 (2000)

4 P.A. Norreys, A.P. Fews, F.N. Beg: Plasma Phys. Controlled Fu- 
sion 40, 175 (1998); G. Pretzler, A. Saemann, A. Pukhov: Phys. Rev. E 58, 1165 (1998); T. Ditmire: Nature 398, 489 (1999); G.S. Sarkisov, V.Yu. Bychenkov, V.T. Tikhonchuk: JETP Lett. 69, 20 (1999)

5 K. Nemoto, A. Maksimchuk, S. Banerjee, K. Flippo, G. Mourou, D. Umstadter, V.Y. Bychenkov: Appl. Phys. Lett. 78, 595 (2001)

6 V.Y. Bychenkov, V.T. Tikhonchuk, S.V. Tolokonnikov: JETP 88, 1137 (1999)

7 V.S. Khoroshkov, E.I. Minakova: Eur. J. Phys. 19, 523 (1998)

8 S.V. Bulanov, T.Zh. Esirkepov, F. Califano, Y. Kato, T.V. Liseikina, K. Mima, N.M. Naumova, K. Nishihara, F. Pegoraro, H. Ruhl, Y. Sentoku, Y. Ueshima: JETP Lett. 71, 407 (2000)

9 B.A. Remington, R.P. Drake, H. Takabe: Phys. Plasmas 7, 1641 (2000)

10 V.Y. Bychenkov, W. Rozmus, A. Maksimchuk, D. Umstadter, C.E. Capjack: Fast ignitor concept with light ions. Plasma Phys. Rep. 27, 1017 (2001)

11 S.J. Gitomer, R.D. Jones, F. Begay, A.W. Ehler, J.F. Kerhart, R. Kristal: Phys. Fluids 29, 2679 (1986)

12 W. Yu, V. Bychenkov, Y. Sentoku, M.Y. Yu, Z.M. Sheng, K. Mima: Phys. Rev. Lett. 85, 570 (2000)

13 K. Krushelnick, E.L. Clark, M. Zepf, J.R. Davies, F.N. Beg, A. Machacek, M.I.K. Santala, M. Tatarakis, I. Watts, P.A. Norreys, A.E. Dangor: Phys. Plasmas 7, 2055 (2000); E.L. Clark, K. Krushelnick, J.R. Davies, M. Zepf, M. Tatarakis, F.N. Beg, A. Machacek, P.A. Norreys, M.I.K. San-tala, I. Watts, A.E. Dangor: Phys. Rev. Lett. 84, 670 (2000)

14 Y. Sentoku, T.V. Liseikina, T.Zh. Esirkepov, F. Califano, N.M. Naumova, Y.Ueshima, V.A.Vshivkov, Y.Kato, K. Mima, K.Nishihara, F. Pegoraro, S.V. Bulanov: Phys. Rev. E 62, 7271 (2000)

15 C.D. Decker, W.B. Mori, T. Katsouleas: Phys. Rev. E 50, 3338 (1994)

16 S.C. Wilks, W.L. Kruer: IEEE J. Quantum Electron. 33, 1954 (1997)

17 T. Tajima, J.M. Dawson: Phys. Rev. Lett. 43, 267 (1979)

18 S.C. Wilks, W.L. Kruer, M. Tabak, A.B. Langdon: Phys. Rev. Lett. 69, 1383 (1992)

19 W.L. Kruer, K. Estabrook: Phys. Fluids 28, 430 (1985)
20 F. Brunel: Phys. Rev. Lett. 59, 52 (1987)

21 E. Esarey, C.B. Schroeder, W.P. Leemans, B. Hafizi: Phys. Plasmas 6, 2262 (1999)

22 A. Pukhov, Z.-M. Sheng, J. Meyer-ter-Vehn: Phys. Plasmas 6, 2847 (1999)

23 A.J. MacKinnon, M. Borghesi, S. Hatchett, M.H. Key, P.K. Patel, H. Campbell, A. Schiavi, R. Snavely, S.C. Wilks, O. Willi: Phys. Rev. Lett. 86, 1769 (2001)

24 L. Gremillet, F. Amiranoff, S.D. Baton, J.C. Gauthier, M. Koening, E. Martinolli, F. Pisani, G. Bonnaud, C. Lebourg, C. Rousseaux, C. Toupin, A. Antonicci, D. Batani, A. Bernardinello, T. Hall, D. Scott, P. Norreys, H. Bandulet, H. Pepin: Phys. Rev. Lett. 83, 5015 (1999)

25 Z. Jiang, J.C. Kieffer, J.P. Matte, M. Chaker, O. Peyrusse, D. Gilles, G. Korn, A. Maksimchuk, S. Coe, G. Mourou: Phys. Plasmas 2, 1702 (1995)

26 A.R. Bell, J.R. Davies, S. Guerin, H. Ruhl: Plasma Phys. Controlled Fusion 39, 653 (1997)

27 J.R. Davies, A.R. Bell, M.G. Haines, S.M. Guerin: Phys. Rev. E 56, 7193 (1997); J.R. Davies, A.R. Bell, M. Tatarakis: Phys. Rev. E 59, 6032 (1999)

28 F. Pisani, A. Bernardinello, D. Batani, A. Antonicci, E. Martinolli, M. Koening, L. Gremillet, F. Amiranoff, S. Baton, J. Davies, T. Hall, D. Scott, P. Norreys, A. Djaoui, C. Rousseaux, P. Fews, H. Bandulet, H. Pepin: Phys. Rev. E 62, 5927 (2000)

29 S.C. Wilks, A.B. Langdon, T.E. Cowan, M. Roth, M. Singh, S. Hatchett, M.H. Key, D. Pennington, A.J. MacKinnon, R.A. Snavely: Phys. Plasmas 8, 542 (2001)

30 A.J. Lichtenberg, M.A. Lieberman: Regular and Stochastic Motion (Springer, New York 1984) Section 5.2

31 V.A. Buts, A.V. Buts: JETP 83, 449 (1996)

32 L.D. Landau, E.M. Lifshitz: The Classical Theory of Fields (Pergamon, Oxford 1975) p. 118

33 G.S. Sarkisov, V.Y. Bychenkov, V.N. Novikov, V.T. Tikhonchuk, A. Maksimchuk, S.Y. Chen, R. Wagner, G. Mourou, D. Umstadter: Phys. Rev. E 59, 7042 (1999)

34 A. Pukhov, Z.M. Sheng, J. Meyer-ter-Vehn: Phys. Plasmas 6, 2847 (1999) 35 K.-C. Tzeng, W.B. Mori, T. Katsouleas: Phys. Rev. Lett. 79, 5258 (1997) 